\title{
Cardiopulmonary exercise testing following bilateral thoracoscopic sympathicolysis in patients with essential hyperhidrosis
}

\author{
Marc Noppen, Patrick Herregodts, Paul Dendale, Jean D'Haens, Walter Vincken
}

\begin{abstract}
Background - Essential hyperhidrosis is characterised by an overactivity of the sympathetic fibres passing through the upper dorsal sympathetic ganglia D2-D3. Anatomical interruption at the D2-D3 level is a highly effective treatment for essential hyperhidrosis but also causes (partial) cardiac denervation and, after surgical sympathicolysis, important impairment of cardiopulmonary exercise function has been observed. The purpose of this study was to compare the results of cardiopulmonary exercise testing between patients with essential hyperhidrosis and a normal control population, and to examine the effects of thoracoscopic D2-D3 sympathicolysis on cardiopulmonary exercise capacity in patients with essential hyperhidrosis.
\end{abstract}

Methods - Maximal, symptom limited incremental exercise tests were performed in 26 patients with severe essential hyperhidrosis one week before and one month after D2-D3 thoracoscopic sympathicolysis, and in 14 age and sex matched healthy volunteers. D2-D3 thoracosopic sympathicolysis was performed using a simplified one stage bilateral procedure. Results - Palmar hyperhidrosis was relieved in every patient, confirming the D2-D3 denervation. A higher peak heart rate $(7 \%)$ was seen in the patient group than in the normal subjects, but all other cardiovascular, metabolic, and respiratory parameters were similar. After D2-D3 thorascopic sympathicolysis, heart rate at rest $(13 \%)$ and at peak exercise $(7 \%)$ were reduced, together with an increase in oxygen pulse. All other parameters remained unchanged.

Conclusions - Sympathetic overactivity relevant to cardiovascular function in essential hyperhidrosis is evident only during sympathetic stimulation. D2-D3 thoracoscopic sympathicolysis causes a small and asymptomatic reduction in maximal and resting heart rate and is not associated with a decrease in exercise capacity, in contrast with the detrimental effects on exercise capacity of open surgical sympathectomy.

(Thorax 1995;50:1097-1100)

Keywords: exercise testing, essential hyperhidrosis, thoracoscopy, sympathicolysis, autonomic function.
Essential hyperhidrosis is characterised by excessive sweating of the palms of the hands and often the soles of the feet and the armpits. ${ }^{1-4}$ The exact cause is unknown, although overactivity of the sympathetic fibres which pass through the dorsal sympathetic ganglia D2 (and D3) has been noted. ${ }^{56}$ Interruption of the sympathetic chain at the D2-D3 level (preferably by thoracoscopic intervention) is an effective and safe treatment for essential hyperhidrosis refractory to conventional local, systemic, or other treatment. ${ }^{4-9}$ However, the D2 and D3 ganglia are also in the direct pathway of sympathetic innervation of the heart. ${ }^{10}$ Interruption of the upper dorsal sympathetic chain has been suggested as treatment for several cardiac disorders - for example, angina pectoris, ventricular tachycardia, and arrythmias associated with the long QT syndrome. ${ }^{11}$

Surgical D2-D3 sympathectomy for essential hyperhidrosis induces important cardiopulmonary impairment. After surgical excision of the D2-D3 ganglia via the supraclavicular approach, $30 \%$ of patients were unable to perform a maximal exercise test due to dyspnoea, fatigue, or dizziness. In order to study the autonomic (dys)function relative to cardiopulmonary function in patients with essential hyperhidrosis, and to study the effects of upper dorsal thoracoscopic sympathicolysis on cardiopulmonary function during exercise, exercise testing was performed on 26 patients with essential hyperhidrosis before and one month after bilateral D2-D3 thoracoscopic sympathicolysis, and in 14 age and sex matched healthy volunteers.

\section{Methods}

PATIENTS

Twenty six patients ( 22 women) with essential hyperhidrosis of mean (SD) age $27(8 \cdot 7)$ years (range 14-44 years) were studied. All suffered from severe hyperhidrosis of the hands and some the feet $(82 \%)$ and armpits $(65 \%)$, which was refractory to local, systemic and/or iontophoretic treatment. Physical examination was otherwise normal, and there was no history of cardiovascular or pulmonary disease. No patient was treated with cardiopulmonary medication. All patients gave informed consent to the study. Patients were studied one week before and one month after sympathicolysis. Fourteen healthy volunteers (11 women) of mean (SD) age $31(5 \cdot 8)$ years (range 22-40) \\ Accepted for publication}


Table 1 Mean (SD) characteristics of controls $(n=14)$ and patients before sympathicolysis $(n=26)$

\begin{tabular}{|c|c|c|}
\hline & Controls & Patients \\
\hline $\mathrm{M}: \mathrm{F}$ & $3: 11$ & $4: 22$ \\
\hline Age (years) & $31(5 \cdot 8)$ & $27(8 \cdot 7)$ \\
\hline Duration (minutes) & $8(2 \cdot 6)$ & $7 \cdot 1(1 \cdot 6)$ \\
\hline Work of peak exercise (watts) & $189 \cdot 6(103 \cdot 6)$ & $186 \cdot 4(49 \cdot 2)$ \\
\hline $\mathrm{Vo}_{2}$ peak $(\mathrm{ml} / \mathrm{kg} / \mathrm{min})$ & $31(11 \cdot 8)$ & $30 \cdot 8(7)$ \\
\hline VE peak $(1 / \mathrm{min})$ & $78.4(25.4)$ & $71 \cdot 2(18 \cdot 8)$ \\
\hline$\Delta \mathrm{VD} / \mathrm{VT}(\Delta \%)$ & $17 \cdot 6(4 \cdot 8)$ & $18.9(7 \cdot 2)$ \\
\hline VT (ml) & $582 \cdot 9(172 \cdot 3)$ & $681.9(304 \cdot 4)$ \\
\hline VT peak (ml) & $2243.6(514.8)$ & $2212.9(593.6)$ \\
\hline$R R$ rest (per minute) & $16 \cdot 8(4 \cdot 6)$ & $17 \cdot 5(3 \cdot 7)$ \\
\hline$R R$ peak (per minute) & $35 \cdot 4(8 \cdot 6)$ & $33 \cdot 5(5 \cdot 3)$ \\
\hline HR rest (per minute) & $81(8 \cdot 7)$ & $89 \cdot 2(16 \cdot 1)$ \\
\hline HR peak (per minute) & $168 \cdot 3(15 \cdot 9)$ & $180.6(12.9)^{*}$ \\
\hline (\% pred) & $94.4(7 \cdot 08)$ & $96 \cdot 6(6.4)$ \\
\hline Slope $\mathrm{HR} / \mathrm{Vo}_{2} / \mathrm{kg}(\mathrm{bpm} / \mathrm{ml} / \mathrm{kg})$ & $3.38(0.58)$ & $3.41(0.69)$ \\
\hline $\mathrm{O}_{2}$ pulse rest $(\mathrm{ml} / \mathrm{beat})$ & $2 \cdot 8(1 \cdot 2)$ & $2 \cdot 2(0 \cdot 9)$ \\
\hline $\mathrm{O}_{2}$ pulse peak ( $\mathrm{ml} /$ beat) & $12 \cdot 2(5 \cdot 8)$ & $10 \cdot 3(3.0)$ \\
\hline $\mathrm{SBP}$ rest $(\mathrm{mm} \mathrm{Hg})$ & $118 \cdot 1(14 \cdot 1)$ & $114 \cdot 2(12 \cdot 7)$ \\
\hline SBP peak $(\mathrm{mm} \mathrm{Hg})$ & $150(14 \cdot 8)$ & $155 \cdot 6(19 \cdot 5)$ \\
\hline DBP rest $(\mathrm{mm} \mathrm{Hg})$ & $76 \cdot 3(4 \cdot 8)$ & $73 \cdot 7(7 \cdot 7)$ \\
\hline DBP peak $(\mathrm{mm} \mathrm{Hg})$ & $82 \cdot 7(10 \cdot 1)$ & $81 \cdot 7(11 \cdot 8)$ \\
\hline
\end{tabular}

$\dot{\mathrm{Vo}}_{2}$ peak $=$ oxygen uptake at peak exercise; $\dot{\mathrm{VE}}$ peak $=$ peak ventilation; $\Delta \mathrm{VD} / \mathrm{VT}=$ change in dead space ventilation; $\mathrm{VT}=$ tidal volume; $\mathrm{RR}=$ breathing frequency; $\mathrm{HR}=$ heart rate; $\mathrm{O}_{2}$ pulse = oxygen pulse; SBP/DBP = systolic/diastolic blood pressure.

${ }^{*} \mathrm{p}<0.05$.

Table 2 Mean (SD) data before and after sympathicolysis in hyperhidrotic patients $(n=26)$

\begin{tabular}{|c|c|c|}
\hline & Before & After \\
\hline Duration (min) & $7 \cdot 1(1 \cdot 6)$ & $7 \cdot 3(1 \cdot 7)$ \\
\hline Work at peak (watts) & $186 \cdot 4(49 \cdot 2)$ & $183.7(48 \cdot 3)$ \\
\hline $\mathrm{Vo}_{2}$ peak $(\mathrm{ml} / \mathrm{min})$ & $1888.5(551 \cdot 6)$ & $1842.5(568.3)$ \\
\hline VE peak $(1 / \mathrm{min})$ & $71 \cdot 2(18 \cdot 8)$ & $73 \cdot 3(17)$ \\
\hline$\Delta \mathrm{VD} / \mathrm{VT}(\Delta \%)$ & $-18.9(7 \cdot 2)$ & $-19 \cdot 9(4 \cdot 8)$ \\
\hline VT at rest $(\mathrm{ml})$ & $681.9(304 \cdot 2)$ & $595(138 \cdot 6)$ \\
\hline VT peak (ml) & $2169.9(583.9)$ & $2119(533.7)$ \\
\hline$R R$ rest (per minute) & $17 \cdot 5(3 \cdot 7)$ & $16 \cdot 4(2 \cdot 8)$ \\
\hline$R R$ peak (per minute) & $33.5(5 \cdot 3)$ & $34.9(5 \cdot 7)$ \\
\hline HR rest (per minute) & $89 \cdot 2(16 \cdot 1)$ & $77 \cdot 4(12 \cdot 5)^{*}$ \\
\hline HR peak (per minute) & $180.6(12.9)$ & $167 \cdot 7(14 \cdot 1)^{*}$ \\
\hline Slope $\mathrm{HR} / \mathrm{Vo}_{2} / \mathrm{kg}(\mathrm{bpm} / \mathrm{ml} / \mathrm{kg})$ & $3.41(0.69)$ & $3.33(0.85)$ \\
\hline $\mathrm{O}_{2}$ pulse rest (mi/beat) & $2 \cdot 19(0.9)$ & $2.3(0.9)$ \\
\hline $\mathrm{O}_{2}$ pulse peak (ml/beat) & $10 \cdot 3(3 \cdot 0)$ & $10.9(3.3)^{*}$ \\
\hline SBP rest $(\mathrm{mm} \mathrm{Hg})$ & $114 \cdot 2(12 \cdot 7)$ & $113 \cdot 4(18 \cdot 3)$ \\
\hline SBP peak $(\mathrm{mm} \mathrm{Hg})$ & $155.6(19.5)$ & $149.6(16.9)$ \\
\hline DBP rest $(\mathrm{mm} \mathrm{Hg})$ & $73 \cdot 7(7 \cdot 7)$ & $72 \cdot 6(5 \cdot 8)$ \\
\hline DBP peak (mm Hg) & $81 \cdot 7(11 \cdot 8)$ & $77 \cdot 2(9.9)$ \\
\hline
\end{tabular}

$\dot{\mathrm{VO}}_{2}$ peak $=$ oxygen uptake at peak exercise; $\mathrm{VE}$ peak = peak ventilation, $\Delta \mathrm{VD} / \mathrm{VT}=$ change in dead space ventilation; $\mathrm{VT}=$ tidal volume, $\mathrm{RR}=$ breathing frequency, $\mathrm{HR}=$ heart rate, $\mathrm{O}_{2}$ pulse = oxygen pulse, $\mathrm{SBP} / \mathrm{DPB}=$ systolic/diastolic blood pressure.

oxygen pulse
$* \mathrm{p}<0.05$ saturation (Sensor Medics oximeter) were monitored continuously and stored on-line. Blood pressure was measured every minute before, during, and up to 15 minutes after exercise with a manual sphygmomanometer. Every subject was allowed an adaptation time of about five minutes, as well as an active warmup (freewheeling) period of 3-4 minutes.

Breath-by-breath gas analysis was performed using a zirconium oxygen analyser and an infrared absorption carbon dioxide analyser which were calibrated daily. Air flows were measured with a thermal conductivity mass flow meter which was calibrated before every test. All data were continuously recorded, stored, and analysed by the 2900 Sensor Medics software package. The parameters measured are given in the results section and shown in the tables.

\section{THORACOSCOPIC SYMPATHICOLYSIS}

All interventions were performed under strict aseptic conditions in a fully equipped operating theatre, using general anaesthesia. Intubation was performed with a Hi-Lo jet endotracheal tube (Mallinckrodt) and ventilation was assured using high frequency jet ventilation (Acutronic) on both lungs.

After creation of a right pneumothorax a $7 \mathrm{~mm}$ trocar was introduced into the pleural cavity through the second intercostal space. The thoracoscope (Richard Wolf, Knitlingen, Germany) was introduced via the trocar, and the dorsal sympathetic chain was identified. An insulated biopsy forceps, connected to a unipolar coagulation unit, was then introduced via a second trocar, under direct vision. The sympathetic trunk was identified and transected at the second and third dorsal rib heads, using 5-15 bursts of 60 watts. After re-expansion of the pneumothorax and skin closure, the same procedure was performed on the other side. Mean duration of this bilateral procedure was about 25 minutes (range 20-40).

to participate as a control population. Patient and control subject characteristics are listed in table 1.

\section{STUDY DESIGN}

Cardiopulmonary exercise testing was performed on a Sensor Medics 2900 Metabolic Measurement Cart system coupled to an ergoline cyclo ergometer (Sensor Medics, Bilthoven, The Netherlands) using a maximal, symptom limited incremental exercise testing protocol consisting of one minute stages with increments of power of 15-20 watt (women) or 25-30 watt (men) every minute. ${ }^{12}$ Before exercise testing baseline spirometric measurements were performed for forced expiratory volume in one second $\left(\mathrm{FEV}_{1}\right)$ and vital capacity (VC), and to calculate maximal voluntary ventilation (MVV $=35 \times \mathrm{FEV}_{1}$ expressed in $1 / \mathrm{min}$ ). Patients breathed through a tight fitting face mask (Survivair Series 7910) equipped with a Rudolph valve (Klaus Rudolph, Kansas City, USA). The 12 lead electrocardiogram (Cardiovit CS-6/12 ECG apparatus, Schiller AG, Baar, Switzerland) and transcutaneous oxygen

\section{STATISTICAL ANALYSIS}

Data are expressed as mean (SD) values. Comparisons between healthy controls and patients before sympathicolysis were made using the Mann-Whitney test. Data before and after sympathicolysis in the patients with essential hyperhidrosis were compared using the Wilcoxon rank sum test. The Fisher exact test was used for comparison of sex ratios. $p$ values of $<0.05$ were considered statistically significant.

\section{Results}

In all patients, palmar hyperhidrosis was relieved by the intervention, confirming complete D2-D3 denervation. All control subjects and all patients successfully completed a maximal exercise test before and after sympathicolysis and the results are summarised in tables 1 and 2.

In basal resting conditions there were no significant differences between controls and patients before treatment, although heart rate and oxygen pulse tended to be lower in the 
patients (table 1). Peak exercise heart rate was significantly higher in the patients before treatment than in the controls: $180.6(12.9)$ versus $168.3(15.9) \mathrm{bpm}(\mathrm{p}<0.05)$, although this difference did not reach statistical significance when expressed as percentage predicted. There were no differences in cardiovascular (blood pressure), ventilatory or metabolic parameters, nor in the subjective reasons for cessation of exercise.

Pairwise data analysis in the patients before and after sympathicolysis showed a significant decrease in resting and maximal heart rate, and an increase in oxygen pulse. These postoperative values are not statistically different from the data obtained in normal volunteers. All other parameters, including the subjective reasons for cessation of exercise, remained unchanged after sympathicolysis.

\section{Discussion}

We have compared cardiopulmonary exercise testing in healthy controls and hyperhidrotic patients, and in these patients before and after thoracoscopic D2-D3 sympathicolysis. Thoracoscopic sympathicolysis had no influence on exercise capacity nor on the ventilatory response to exercise, and only minimal effects on the cardiac response to exercise.

Essential hyperhidrosis is probably caused by increased activity of the sympathetic fibres which pass through the D2 and D3 dorsal sympathetic ganglia. It causes autonomic dysfunction with less reflex bradycardia in response to the Valsalva manoeuvre or facial immersion, a higher degree of cutaneous vasoconstriction in response to finger (or cold) immersion, ${ }^{5}$ and a reduction in cholinomimetic-induced hypothermia. $^{6}$

Our results suggest that the overactivity of the sympathetic nervous system in the patients has a very small effect only on the integrated cardiorespiratory metabolic function at rest, and a moderate effect at maximal exercise. This is in agreement with the fact that, in the resting state (when the stress on the cardiovascular system is minimal), heart rate is mainly under vagal restraint, ${ }^{13}$ whereas during exercise an increase in heart rate occurs through a simultaneous decrease in vagal activity and an increase in sympathetic activity. ${ }^{13-15}$ The sympathetic overactivity in patients with essential hyperhidrosis therefore seems to exert its effect mainly in conditions of sympathetic activation.

After sympathicolysis the heart rate at rest was significantly reduced, confirming that in these patients resting heart rate is (at least partially) also under sympathetic control. More important changes have been reported by Papa et $a l^{16}$ who observed a decrease of $16 \%$ in resting heart rate and $18 \%$ in maximal heart rate after bilateral surgical (supraclavicular approach) D2 and D3 sympathectomy. Furthermore, about $30 \%$ of their patients were unable to complete the postoperative exercise test because of weakness, dyspnoea, dizziness, or general fatigue at submaximal levels of work. These dramatic changes were not seen in our patients, probably because thoracoscopic sym- pathicolysis offers a far more accurate and precise interruption of the sympathetic chain ${ }^{4}$ and because the procedure causes less effect on lung volumes and airflow rates than conventional surgery. ${ }^{17} 18$

Our findings are comparable with the effects of sympathectomy performed in patients with various cardiovascular disorders - for example, paroxysmal atrial tachycardia, angina, and hypertension. Bilateral D2-D5 sympathectomy decreasing the heart rate from $8 \%$ to $28.9 \%$ at rest and from $21.8 \%$ to $31.9 \%$ during exercise have been observed. ${ }^{16}{ }^{19}$ Similarly, after medical $\beta$ receptor blockade only small decreases in heart rate occur at rest, but greater reductions occur at maximal exercise. ${ }^{151920}$ After $\beta$ receptor blockade cardiac output is maintained by a parallel increase in stroke volume through the Frank-Starling mechanism ${ }^{21}$ which may explain the increase in oxygen pulse seen in our patients after sympathicolysis. Finally, similar findings have been observed after acute epidural sympathetic denervation by a sensory block of C4-D7: mean resting heart rate significantly dropped from 66 to 58 beats per minute. ${ }^{22}$

D2-D3 sympathicolysis in patients with essential hyperhidrosis had no effect on ventilatory and metabolic parameters, in constrast to the effects of $\beta$ blockade in normal subjects in whom measured oxygen uptake is slightly reduced by about $5 \%$, and the anaerobic threshold is lowered by about $20 \% .^{20}$ The authors would like to thank the nursing staff of the Re-
spiratory Division (Karine Michiels, Anne Ringoir, Brigitte spiratory Division (Karine Michiels, Anne Ringoir, Brigitte
Terryn, Bea Van Elewijck, Sonja Van Poyer, Daniel Schuermans) Terryn, Bea Van Elewijck, Sonja Van Poyer, Daniel Schuermans)
for their enthusiasm and competence and Hilde De Smedt for the preparation of the manuscript.

1 Cloward R. Hyperhidrosis. $\mathcal{F}$ Neurosurg 1969;30:545-51.

2 Harris JD, Jepson RP. Essential hyperhidrosis. Med $\mathcal{f}$ Aust 1971;2:135-8.

3 Adar R, Kurchin A, Zweig A, Mozes M. Palmar hyperhidrosis and its surgical treatment. A report of 100 cases. Ann Surg 1977;186:34-41.

4 Noppen M, Herregodts P, D'Haens J, Vincken W. ThoNoppen $M$, Herregodts $P$, D'Haens J, Vincken W. Tho-
racoscopic sympathicolysis for essential hyperhidrosis: results in 35 patients and review of the literature. Chest (submitted).

5 Shih CJ, Wu JJ, Lin MT. Autonomic dysfunction in palmar hyperhydrosis. F Autonomic Nerv Syst 1983;8:33-43.

6 Shih CJ, Lin MT. Effects of cholinomimetic drugs on sudomotor, metabolic, respiratory, vasomotor, and temperature response in palmar hyperhidrosis. $\mathcal{F}$ Neurosurg 1980;53:684-9.

7 Byrne J, Walsch TN, Hederman WP. Endoscopic transthoracic electrocautery of the sympathetic chain for palma

8 Claes G, Göthberg C. Endoscopic transthoracic electrocautery of the sympathetic chain for palmar and axillary
thecicic trocautery of the sympathetic chain for
hyperhidrosis. Brf $\mathcal{F}$ Surg 1991;78:760.

9 Lin CC. Extended thorascopic T2-sympathectomy in treatment of hyperhidrosis: experience with 130 consecutive cases. $\mathcal{f}$ Laparoendosc Surg 1992;2:1-6.

10 Firestone $\mathrm{L}$. Autonomic influences on cardiac function: lessons from the transplanted (denervated) heart. Int Anaesthesiol Clin 1989;27:283-91.

11 Kadowaki MH, Levitt JM. Sympathectomy in the treatment of angina and arrhythmias. Ann Thorac Surg 1986;41: $572-8$.

12 Zabellos RJ, Weisman IM. Behind the scenes of cardiopulmonary exercise testing. Clin Chest Med 1994;15:193213.

13 Shephard JT. Circulatory response to beta-adrenergic blockade at rest and during exercise. Am $\mathcal{f}$ Cardiol 1985;55: 87D-94D

14 Savin WM, Davidson DM, Haskell WL. Autonomic contribution to heart rate recovery from exercise in humans. f Appl Physiol 1982;53:1572-5.

15 Epstein SE, Robinson BF, Kahler RL, Braunwald E. Effects of beta-adrenergic blockade on the cardiac response to maximal and submaximal exercise in man. $\mathcal{F}$ Clin Invest 1965;44:1745-53.

16 Papa MZ, Schneiderman J, Tucker E, Bass A, Drori Y, Adar R. Cardiovascular changes after bilateral upper dorsal sympathectomy. Ann Surg 1986;204:715-8.

17 Molho M, Kurchin A, Ohry A, Adar R. Pulmonary function 
abnormality after upper dorsal sympathectomy. $A m$ Rev Respir Dis 1977;116:879-83.

18 Molho M, Shemesh E, Gordon D, Adar R. Pulmonary functional abnormality after upper dorsal sympathectomy: a comparison between the supraclavicular and transaxillary approach. Chest 1980;77:651-5.

19 Swithwick RH, Chapman EM, Kinsey D, Whitelaw GP

The human heart rate: some observations and deductions based upon the effect of removing portions of the sympathetic nervous system in man. Surgery 1949;26:727-34. 20 Petersen ES, Whipp BJ, Davis JA, Huntsman DJ, Brown
HV, Wasserman K. Effects of beta-adenergic blockade on ventilation and gas exchange during exercise in humans. f Appl Physiol 1983;54:1306-13.

21 Anderson K, Vik-Mo H. Role of the Frank-Starling mechanism during maximal semisupine exercise after oral atenolol. Br Heart f 1982;48:149-55.

22 Takeshima S, Dohi S. Circulatory responses to baroreflexes, valsalva manoeuvre, coughing, swallowing and nasal stimulation during acute cardiac sympathectomy by epistimulation during acute cardiac sympathectomy by epi500-8. 